\title{
Impact of chronic co-infection in pulmonary Mycobacterium avium complex disease after treatment initiation
}

Naohisa Urabe

Toho University Omori Medical Center

Susumu Sakamoto ( $\nabla$ susumu1029@gmail.com )

Toho University Omori Medical Center

Yui Shimanuki

Toho University Omori Medical Center

Takumi Kanokogi

Toho University Omori Medical Center

Takumi Motohashi

Toho University Omori Medical Center

Nanami Anzai

Toho University Omori Medical Center

Chiaki Kato

Toho University Omori Medical Center

Asuka Yamaguchi

Toho University Omori Medical Center

Nozomi Tokita

Toho University Omori Medical Center

Sakae Homma

Toho University Omori Medical Center

Kazuma Kishi

Toho University Omori Medical Center

\section{Research Article}

Keywords: Nontuberculous mycobacterium, Mycobacterium avium complex, Co-infection, Pseudomonas aeruginosa

Posted Date: January 31st, 2022

DOI: https://doi.org/10.21203/rs.3.rs-1253953/v1 
License: (c) (i) This work is licensed under a Creative Commons Attribution 4.0 International License. Read Full License 


\section{Abstract}

Background: The impact of co-infection with other pathogenic microorganisms after initiation of treatment for pulmonary Mycobacterium avium complex (MAC) disease has not been clearly demonstrated. This study sought to clarify clinical outcomes of co-infection with MAC

after antimycobacterial therapy for MAC.

Methods: Co-infection status was designated as the detection of pathogenic microorganisms other than MAC in at least two consecutive sputum cultures 6-24 months after initiation of treatment. Chest computed tomography (CT) findings and culture results were compared between co-infection and MAC alone groups.

Results: The co-infection and MAC alone groups comprised 12 and 36 patients, respectively. The proportion of patients with sputum culture positive for MAC after 24 months of therapy did not differ significantly between the two groups [25\% (3/12) vs. $16.7 \%(6 / 36) ; p=0.671]$. The proportion of patients with improved chest CT score after 24 months of starting treatment compared to baseline was significantly lower for the co-infection group than for the MAC alone group [16.7\% (2/12) vs. $79.4 \%$ (27/34); $p<0.001]$. In the co-infection group, mean CT score values at 12 and 24 months did not differ from baseline. However, the MAC alone group showed significant improvement at 12 and 24 months compared with baseline.

Conclusions: Co-infection with other pathogenic microorganisms has no effect on the therapeutic efficacy of MAC but interferes with improvement in chest CT findings.

\section{Background}

Pulmonary nontuberculous mycobacteria (NTM) disease is increasing in incidence worldwide and has become an important concern [1]. In Japan, the incidence of pulmonary NTM disease has been gradually increasing and exceeded the incidence of mycobacterial tuberculosis for the first time in 2014 [2]. The type of NTM differs by region. In Japan, Mycobacterium avium complex (MAC) accounts for about $90 \%$ of the total [2]. Pulmonary MAC disease is a chronic lower respiratory tract infection caused by MAC. Chronic lower respiratory tract infections are a burden because of the difficulty with identifying the causative microorganism; co-infections with multiple microorganisms have also been reported [3]. In a previous study we report that $12.2 \%$ of patients with pulmonary MAC disease had co-infections with Haemophilus influenzae (H. influenzae) and Pseudomonas aeruginosa (P. aeruginosa) [4]. According to Fujita et al., $45.1 \%$ of patients with pulmonary MAC disease had chronic co-infection with other pathogenic microorganisms, and chronic $P$. aeruginosa co-infection increased after the initiation of treatment for pulmonary MAC disease [5]. However, the impact of co-infection with other pathogenic microorganisms after initiation of treatment for pulmonary MAC disease has not been clarified. 
Thus, we investigated the impact of co-infection with other pathogenic microorganisms after initiation of treatment for pulmonary MAC disease.

\section{Methods}

\section{Study design}

This single-center retrospective cohort study included 48 patients with pulmonary MAC disease who had started treatment during the period from November 2014 through February 2019 at Toho University Omori Medical Center. All patients were required to fulfill the American Thoracic Society (ATS) criteria for the diagnosis of NTM [6], and they continued MAC treatment from baseline for at least 12 months (46 patients continued for at least 24 months; 2 patients continued for at least 12 months). Sputum culture for mycobacteria, other bacteria, and fungi was performed at least 3 times during the 6-24 months after treatment initiation for pulmonary MAC disease. Patients who had difficulty with sputum collection were excluded.

\section{Definition of co-infection and treatment failure in pulmonary MAC disease}

We defined co-infection as the detection of pathogenic microorganisms other than MAC in at least two consecutive sputum cultures 6-24 months after initiation of treatment for pulmonary MAC disease. At baseline, co-infection was defined as detection of pathogenic microorganisms other than MAC in at least two separate sputum cultures or at least one bronchoalveolar lavage. Co-infection at baseline was designated 'base co-infection'. Treatment success was defined as sputum culture conversion to MAC negative within 12 months of treatment. Treatment failure was designated in patients with persistent MAC detection in sputum culture after 12 months of treatment

We compared the results of high-resolution CT (HRCT) of the chest, microbiological tests, and subjective symptoms between the co-infection and MAC alone groups. Univariate and multivariate logistic regression analysis was also performed to identify factors independently associated with co-infection.

\section{Data collection}

The following patient data were collected: age, sex, body mass index (BMI), smoking history, serum antiglycopeptidolipid-core IgA titer, comorbidities, chest and paranasal sinuses CT, and sputum and bronchoscopy culture results. Most patients underwent Chronic Obstructive Pulmonary Disease (COPD) assessment testing (CAT) to confirm subjective symptoms at the time of treatment initiation and at 6,12 , 18 , and 24 months thereafter [7].

\section{Chest CT score}

Chest HRCT score was reviewed as previously described with slight modification as follows [8]. We divided the lungs into 6 zones at the levels of the carina and inferior pulmonary vein. Using HRCT, we 
categorized the 4 types of pulmonary lesions in MAC (cavities, bronchiectasis, nodules, and infiltration) into 5 stages, according to the occupation area in each section (0: no lesion, $1: 1-24 \%$ occupied, 2: 2549\% occupied, 3: $50-74 \%$ occupied, and 4: $75-100 \%$ occupied). In addition, changes in cavity wall thickness were reflected by assigning +2 points for cavity wall thickening by more than $1 \mathrm{~mm}$ and -2 points for thinning (Figure, 1). Two respiratory physicians with over 13 years of experience reviewed Chest CT scores independently. Improved Chest CT score was defined as a decrease. We investigated the relationship of co-infection, treatment failure, and serial changes in chest CT findings.

\section{Statistical analysis}

Data are presented as the number of patients and percentages. Age, BMl, chest CT score, and CAT score are expressed as mean \pm SD. Associations of categorical and continuous variables between patients in the co-infection and MAC alone groups were tested with the chi-squared or Fisher's exact test, and the Mann-Whitney $\mathrm{U}$ test, respectively. Factors independently associated with co-infection were evaluated with univariate and multivariate logistic regression. Multivariate logistic regression was performed using a stepwise method. A P value of $<0.05$ was considered to indicate statistical significance. Statistical analyses were performed with SPSS software version 22 (IBM Corp., Armonk, NY).

\section{Results}

\section{Clinical characteristics}

In total, 48 patients were included in this study; the co-infection group comprised 12 patients (mean age $72.1 \pm 6.9$ years; 2 men, 10 women) and the MAC alone group included 36 patients (mean age $68.3 \pm 11.8$ years; 7 men, 29 women). The characteristics of all 48 patients are shown in Table 1. At baseline, there were significant differences between the co-infection and MAC alone groups in scores for chest CT (12.6 \pm 3.3 vs. $9.1 \pm 4.1 ; p=0.007)$, bronchiectasis lesions score on chest CT ( $3.7 \pm 1.9$ vs. $2.3 \pm 1.6 ; p=0.035)$, subjective cough symptoms ( $2.5 \pm 1.4$ vs. $1.3 \pm 1.2 ; P=0.015)$, and sputum ( $2.6 \pm 1.8$ vs. $1.1 \pm 1.0 ; p=0.021)$. The proportion of patients with cavitary lesions ( $25 \%$ vs. $50 \%$; $p=0.185)$, cavitary lesions score on chest CT $(0.4 \pm 0.9$ vs. $1.0 \pm 1.5 ; p=0.117)$ and the rate of base co-infection did not differ significantly between the two groups ( $50 \%$ vs. $38.9 \%$; $p=0.52$ ). Both groups showed the same resistance rate to CAM of $8.3 \%$ at 24 months after treatment initiation. Overall treatment success rate for MAC was $81.2 \%$ (39/48) with no significant differences between the co-infection and MAC alone groups ( $75 \%$ vs $83.3 \% ; p=0.671$ ).

\section{Microbiological test results in the co-infection group}

Serial changes in microbiological test results in the co-infection group are shown in Table 2. At baseline, 6 of the 12 patients had base co-infection, namely 2 patients with $H$. influenzae, 2 with Methicillin-sensitive Staphylococcus aureus (MSSA), 1 with P. aeruginosa, and 1 with Escherichia coli (E. coli). In 3 of the 6 patients the same organism was detected in base co-infection and at 12-24 months after treatment for MAC (1 each with E. coli, P. aeruginosa, and MSSA). P. aeruginosa was the most common pathogenic 
microorganism in co-infections after the treatment for MAC, accounting for same in 7 of 12 (58.3\%) patients.

\section{Serial changes in Chest CT score}

Table 3 shows the clinical course in patients with pulmonary MAC disease. In total, CT score was improved in $72.9 \%$ and $63 \%$ of patients at 12 months and 24 months after treatment initiation. At 24 months after treatment, the proportion of patients with improved chest CT score was significantly lower in the co-infection group than the MAC alone group ( $16.7 \%$ vs. $79.4 \%$; $p<0.001)$.

Figure 2 shows serial changes in chest CT score in the 2 groups. In the co-infection group, mean CT score at 12 and 24 months did not differ compared with the baseline CT score $(12.2 \pm 5.3$ and $15.8 \pm 6.0$ vs. 12.6 $\pm 3.3 ; p=0.757$ and $p=0.55$ ). However, in the MAC alone group, mean CT score at 12 and 24 months was significantly improved compared with the baseline score $(5.6 \pm 5.0$ and $5.9 \pm 6.5$ vs. $9.1 \pm 4.1 ; p<0.001$ and $p<0.001)$.

Figure 3 shows serial changes in chest CT score in the MAC alone and treatment success group $(n=30)$; co-infection and treatment success group $(n=9)$; and the treatment failure group $(n=9)$. In the co-infection and treatment success group, mean values at 12 and 24 months did not differ significantly from baseline CT score $(11.9 \pm 5.9$ and $15.8 \pm 6.9$ vs. $12.8 \pm 3.7 ; p=0.604$ and $p=0.196)$. In the treatment failure group, mean values at 12 and 24 months did not differ significantly compared with the baseline CT score (11.4 \pm 3.0 and $13.3 \pm 4.6$ vs. $11.2 \pm 2.6 ; p=0.803$ and $p=0.220$ ). However, the values at 12 and 24 months were significantly better than the baseline CT score $(4.6 \pm 4.8$ and $4.6 \pm 6.0$ vs. $8.7 \pm 4.2 ; p<0.001$ and $\mathrm{p}<0.001)$ in the MAC alone and treatment success group.

\section{Serial changes in CAT score}

At 12 and 24 months after initiation of MAC treatment, the proportion of patients with improved CAT scores did not differ significantly between the co-infection and MAC alone groups ( $70 \%$ vs. $54.8 \%$ and $40 \%$ vs. $48.3 \% ; p=0.48$ and $p=0.726$ ). The proportion of patients with improvement in relation to sputum score also did not differ significantly between the co-infection and MAC alone groups ( $50 \%$ vs. $35.5 \%$ and $20 \%$ vs. $24.1 \% ; p=0.472$ and $p=1)($ Table 3$)$.

Figure 4 shows serial changes in CAT score. The co-infection group showed no significant difference in mean CAT score at 12 and 24 months compared with baseline $(11.6 \pm 9.8$ and $13.8 \pm 10.9$ vs. $11.1 \pm 7.2$; $p=0.576$ and $p=0.131)$; in the MAC alone group the CAT score at 12 and 24 months did not differ significantly from the baseline score $(7.5 \pm 7.0$ and $7.2 \pm 6.8$ vs. $7.9 \pm 6.6 ; p=0.724$ and $p=0.845)$.

Sputum score in CAT score was independently associated with co-infection (Table 4). Figure 5 shows serial changes in sputum score. In the co-infection group, the mean value at 12 and 24 months did not differ significantly compared with the baseline score $(2.3 \pm 1.7$ and $2.7 \pm 1.8$ vs. $2.6 \pm 1.8 ; p=0.565$ and $p=1.0$ ). Also, in the MAC alone group, the mean sputum score at 12 and 24 months, the score did not differ significantly from baseline $(0.97 \pm 1.11$, and $0.97 \pm 0.98$ vs. $1.1 \pm 1.0 ; p=0.536$ and $p=0.345)$. 


\section{Risk factors for co-infection}

Table 4 shows the results of univariate and multivariate logistic regression analysis of independent associations with co-infection. Higher chest CT score (Odds Ratio [OR], 1.27; 95\% confidence interval [Cl], 1.04-1.55; $p=0.017)$, higher sputum score (OR, 2.35; $95 \% \mathrm{Cl}, 1.28-4.31 ; \mathrm{p}=0.006)$, and higher cough score (ORs, $2.2 ; 95 \% \mathrm{Cl}, 1.17-4.14 ; \mathrm{p}=0.014$ ) were independently associated with co-infection in univariate analysis. Higher sputum score (ORs, $2.02 ; 95 \% \mathrm{Cl}, 1.08-3.79 ; \mathrm{p}=0.028)$ was independently associated with co-infection in multivariate analysis.

\section{Discussion}

We demonstrated the impact of co-infection with other pathogenic microorganisms after initiation of treatment for pulmonary MAC disease. Although co-infection with other pathogenic microorganisms does not affect therapeutic efficacy in MAC, these organisms may interfere with improvement of chest CT findings. Few studies have investigated the impact of co-infection with other pathogenic microorganisms in pulmonary MAC disease [5, 9]. Fujita et al. reported chronic co-infection with other pathogenic microorganisms including MSSA, $P$. aeruginosa, and Aspergillus spp. in $45.1 \%$ of patients with pulmonary MAC disease [5]. According to Kamata et al., chronic co-infection with $P$. aeruginosa was seen in $7.8 \%$ of patients with pulmonary MAC disease [9]. It is important to note that these were cross-sectional studies, and so did not clarify the impact of co-infection on the efficacy of MAC treatment. To our knowledge, this is the first report to investigate the impact of co-infection with other pathogenic microorganisms on clinical course after initiation of treatment for pulmonary MAC disease.

This study showed that sputum culture conversion or treatment success rate was $81 \%$, improvement in chest CT score was $63 \%$, and the rate of CAM resistance was $8.3 \%$. These results were consistent with those from previous studies. Earlier reports of macrolide-inclusive daily regimens have shown that the rate of sputum culture conversion was $42-92 \%$ [10-14], chest imaging improvement was $68-82 \%[12,13]$, and macrolide resistance was $9-15 \%[10,11,14]$. In addition, the rates of MAC culture conversion and CAM resistance did not differ significantly between the co-infection and MAC alone groups. These results suggest that co-infection after the initiation of treatment for MAC did not affect the treatment efficacy.

Nevertheless, the proportion of patients with improved chest CT scores was significantly lower in the coinfection group than in the MAC alone group at 24 months after initiation of treatment. Furthermore, serial changes in chest CT score in the co-infection group showed no significant improvement, even after excluding patients with MAC treatment failure. Thus, co-infection with other pathogenic microorganisms does not affect the outcome of MAC treatment but worsens chest CT findings. The worse chest CT findings may have been as a result of other pathogenic microorganisms that gained dominance due to weakening of the competing MAC.

Among 6 of the 12 patients who had base co-infection in the co-infection group, only 3 had the same bacterial species detected until post MAC treatment. Clarithromycin-susceptible bacteria such as MSSA 
and $H$. influenzae decreased after MAC treatment while clarithromycin-resistant bacteria such as $P$. aeruginosa and Nocardia spp. increased after MAC treatment. We speculated that MAC treatment suppressed the proliferation of MAC and other clarithromycin (CAM)-susceptible bacteria, and this might foster a conversion of the bacteria to clarithromycin-resistant. This result is consistent with previous reports showing that $P$. aeruginosa was less frequently isolated from positive MAC sputum cultures and more often isolated after MAC sputum conversion [5].

Previous studies showed that patients with NTM bronchiectasis, including those with cystic fibrosis, had a lower rate of chronic $P$. aeruginosa infection compared with non-NTM infection [15, 16]. MAC and other pathogenic microorganisms, especially $P$. aeruginosa, interact with each other and culture results may reflect the dominant pathogenic species at that time. Therefore, we speculate that the negative MAC culture in the co-infection group may not only be due to the effect of MAC treatment but also due to the suppression of MAC culture by other potentially infectious pathogenic microorganisms that became dominant.

In this study, subjective symptoms were more severe in the co-infection group at baseline. Specifically, high sputum score at baseline was an independent risk factor for co-infection. According to Kamata et al., co-infection with $P$. aeruginosa worsened subjective symptoms in patients with pulmonary MAC [5]. Previous reports showed that $P$. aeruginosa colonization was an independent predictor of hospital admission in bronchiectasis [17]. But in our study, the rate of baseline co-infection showed no significant difference between the co-infection and MAC alone groups. We surmised that the presence of trace amounts of other bacteria undetectable by conventional culture in the co-infection group at baseline was the cause of the severe subjective symptoms, and that the bacteria may have become apparently detectable with MAC treatment.

Several risk factors for developing co-infection in patients with MAC have been reported. This study showed that bronchiectasis score was significantly higher in the co-infection group than the MAC alone group. However, cavitary lesion score and the frequency of concomitant emphysema did not differ between the two groups. In addition, 7 out of 12 patients had $P$. aeruginosa co-infection and none had Aspergillus co-infection. In the Fujita study, of the 124 patients with co-infection, 18 and 35 patients had aspergillus and $P$. aeruginosa infection, respectively. Risk factors for co-infection were reported to be COPD and $M$. intracellulare infection [5]. Patients with $M$. intracellulare infection were more likely to have fibro-cavitary disease than those with $M$. avium infection [18] and cavitary lesions have been reported to be a risk factor for complications of chronic pulmonary aspergillus infection in pulmonary MAC [19]. In contrast, the Kamata study included 19 patients with MAC and $P$. aeruginosa exclusively. The severity of bronchiectasis, but not cavitary lesions, was associated with $P$. aeruginosa co-infection. The disparity in risk factors for co-infection in these studies may be due to the presence or absence of Aspergillus. A previous study showed that severity of bronchiectasis was significantly associated with the presence of chronic $P$. aeruginosa infection in patients with non-cystic fibrosis bronchiectasis [20]. We speculate that patients with cavitary lesions are more likely to have co-infection with Aspergillus, whereas those with 
severe bronchiectasis were more likely to be infected with certain types of bacteria including $P$. aeruginosa.

These findings notwithstanding, this study had several limitations that should be mentioned. Firstly, it was a single-center study in a small number of patients. Thus, the findings may not be generalizable to a larger, more diverse population. Secondly, some patients were excluded from this study due to missing sputum examination and chest CT evaluations. These excluded patients might have had infections from a different type of pathogen. Thirdly, the potential presence of indigenous oral bacterial populations cannot be ruled out, and so this may not accurately reflect the status of the lower airway flora because not all patients underwent bronchoscopy.

\section{Conclusion}

Although co-infection with other pathogenic microorganisms does not affect the therapeutic efficacy in MAC, these organisms may interfere with improvement of chest CT findings.

\section{Abbreviations}

ATS

American Thoracic Society

$\mathrm{BMI}$

body mass index

COPD

Chronic Obstructive Pulmonary Disease

CAT

Chronic Obstructive Pulmonary Disease assessment testing

CAM

clarithromycin

CT

computed tomography

$\mathrm{Cl}$

confidence interval

E. coli

Escherichia coli

H. influenzae

Haemophilus influenzae

HRCT

high-resolution CT

MSSA

Methicillin-sensitive Staphylococcus aureus

MAC 
Mycobacterium avium complex

NTM

Nontuberculous mycobacteria

OR

Odds Ratio

$P$. aeruginosa

Pseudomonas aeruginosa.

\section{Declarations}

\section{Ethics approval and consent to participate}

This study was conducted in accordance with the amended Declaration of Helsinki and the Ethics Committee of Toho University Omori Medical Center approved the protocol (Approval No.M21218). All methods were carried out in accordance with relevant guidelines and regulations. All patients provided written informed consents to participate in the study.

\section{Consent for publication}

Not applicable

\section{Availability of data and materials}

All data generated or analysed during this study are included in this published article [and its supplementary information files].

\section{Conflict of Interest Statement}

The authors have no conflicts of interest to declare.

\section{Competing interests}

The authors declare that they have no competing interests.

\section{Funding Sources}

This study received no funding.

\section{Author Contributions}

NU designed the study, analyzed, and drafted the manuscript, SS and KK designed the study and participated in editing the manuscript, SH confirmed the analyses, and revised the article for intellectual content, YS and TK conceptualized the study and revised the article for intellectual content, TM and NA conceptualized the study, supervised, CK, AY and NT participated in editing the manuscript and revised 
the article for intellectual content. All authors read and critically revised the first as well as the subsequent and final drafts of this manuscript. The author(s) read and approved the final manuscript.

\section{Acknowledgments}

Not applicable.

\section{References}

1. Brode SK, Daley CL, Marras TK. The epidemiologic relationship between tuberculosis and nontuberculous mycobacterial disease: a systematic review. Int J Tuberc Lung Dis. 2014;18 (11): 13707.

2. Namkoong $\mathrm{H}$, Kurashima A, Morimoto $\mathrm{K}$, Hoshino $\mathrm{Y}$, Hasegawa $\mathrm{N}$, Ato $\mathrm{M}$, et al. Epidemiology of Pulmonary Nontuberculous Mycobacterial Disease, Japan. Emerg Infect Dis. 2016; 22 (6): 1116-7.

3. Cox MJ, Turek EM, Hennessy C, Mirza GK, James PL, Coleman M, et al. Longitudinal assessment of sputum microbiome by sequencing of the 16S rRNA gene in non-cystic fibrosis bronchiectasis patients. PLoS One. 2017;12(2): e0170622.

4. Urabe N, Sakamoto S, Sano G, Ito A, Homma S. Characteristics of patients with bronchoscopydiagnosed pulmonary Mycobacterium avium complex infection. J Infect Chemother. 2018; 24 (10): 822-7.

5. Fujita K, Ito Y, Hirai T, Kubo T, Togashi K, Ichiyama S, et al. Prevalence and risk factors for chronic coinfection in pulmonary Mycobacterium avium complex disease. BMJ Open Respir Res. 2014;1(1): e000050.

6. Griffith DE, Aksamit T, Brown-Elliott BA, Catanzaro A, Daley C, Gordin F, et al. An official ATS/IDSA statement: diagnosis, treatment, and prevention of nontuberculous mycobacterial diseases. Am J Respir Crit Care Med. 2007; 175 (4): 367-416.

7. Jones PW, Tabberer M, Chen WH. Creating scenarios of the impact of COPD and their relationship to COPD Assessment Test (CAT ${ }^{T M}$ ) scores. BMC Pulm Med. 2011; 11: 42.

8. Morimoto K, Yoshiyama T, Kurashima A, Sasaki Y, Hoshino Y, Yoshimori K, et al. Nutritional indicators are correlated with the radiological severity score in patients with Mycobacterium avium complex pulmonary disease: a cross-sectional study. Intern Med. 2014; 53 (5): 397-401.

9. Kamata H, Asakura T, Suzuki S, Namkoong H, Yagi K, Funatsu Y, et al. Impact of chronic Pseudomonas aeruginosa infection on health-related quality of life in Mycobacterium avium complex lung disease. BMC Pulm Med. 2017; 17(1): 198.

10. Wallace RJ, Jr., Brown BA, Griffith DE, Girard WM, Murphy DT. Clarithromycin regimens for pulmonary Mycobacterium avium complex. The first 50 patients. Am J Respir Crit Care Med. 1996; 153(6 Pt 1): $1766-72$.

11. Griffith DE, Brown BA, Cegielski P, Murphy DT, Wallace RJ, Jr. Early results (at 6 months) with intermittent clarithromycin-including regimens for lung disease due to Mycobacterium avium 
complex. Clin Infect Dis. 2000; 30 (2): 288-92.

12. Jeong BH, Jeon K, Park HY, Kim SY, Lee KS, Huh HJ, et al. Intermittent antibiotic therapy for nodular bronchiectatic Mycobacterium avium complex lung disease. Am J Respir Crit Care Med. 2015; 191 (1): 96-103.

13. Miwa S, Shirai M, Toyoshima M, Shirai T, Yasuda K, Yokomura K, et al. Efficacy of clarithromycin and ethambutol for Mycobacterium avium complex pulmonary disease. A preliminary study. Ann Am Thorac Soc. 2014; 11 (1): 23-9.

14. Ito Y, Miwa S, Shirai M, Kanai M, Fujita K, Ohba H, et al. Macrolide resistant Mycobacterium avium complex pulmonary disease following clarithromycin and ethambutol combination therapy. Respir Med. 2020; 169: 106025.

15. Binder AM, Adjemian J, Olivier KN, Prevots DR. Epidemiology of nontuberculous mycobacterial infections and associated chronic macrolide use among persons with cystic fibrosis. Am J Respir Crit Care Med. 2013; 188 (7): 807-12.

16. Aksamit TR, O'Donnell AE, Barker A, Olivier KN, Winthrop KL, Daniels MLA, et al. Adult Patients with Bronchiectasis: A First Look at the US Bronchiectasis Research Registry. Chest. 2017; 151 (5): 98292.

17. Chalmers JD, Goeminne P, Aliberti S, McDonnell MJ, Lonni S, Davidson J, et al. The bronchiectasis severity index. An international derivation and validation study. Am J Respir Crit Care Med. 2014; 189(5): 576-85.

18. Koh WJ, Jeong BH, Jeon K, Lee NY, Lee KS, Woo SY, et al. Clinical significance of the differentiation between Mycobacterium avium and Mycobacterium intracellulare in M. avium complex lung disease. Chest. 2012; 142(6): 1482-8.

19. Song JW, Koh WJ, Lee KS, Lee JY, Chung MJ, Kim TS, et al. High-resolution CT findings of Mycobacterium avium-intracellulare complex pulmonary disease: correlation with pulmonary function test results. AJR Am J Roentgenol. 2008; 191 (4): W160.

20. Dimakou K, Triantafillidou C, Toumbis M, Tsikritsaki K, Malagari K, Bakakos P. Non CFbronchiectasis: Aetiologic approach, clinical, radiological, microbiological and functional profile in 277 patients. Respir Med. 2016; 116: 1-7.

\section{Tables}

Tables are available in the Supplemental Files section.

\section{Figures}

\section{Figure 1}


Stages of pulmonary lesions in MAC disease by chest CT score. Cavity wall thickness was assigned +2 points if the thickness increased by more than $1 \mathrm{~mm}$ and -2 points were given for thinning.

\section{Figure 2}

Serial changes in chest CT score. Gray lines indicate change in chest CT score in each patient after initiation of treatment for pulmonary MAC disease. Black lines indicate the mean for all patients. A paired t-test was used to compare values at 12 and 24 months with baseline.

\section{Figure 3}

Gray lines indicate change in Chest CT score in each patient after initiation of treatment for pulmonary MAC diseases. Black lines indicate the mean for all patients. A paired t-test was used to compare values at 12 and 24 months with baseline values.

\section{Figure 4}

Gray lines indicate change in CAT score in each patient after initiation of treatment for pulmonary MAC disease. Black lines indicate the mean for all patients. A paired t-test was used to compare values at 12 and 24 months with baseline values.

\section{Figure 5}

Gray lines indicate change in sputum score in each patient after initiation of treatment for pulmonary MAC disease. Black lines indicate the mean for all patients. A paired t-test was used to compare values at 12 and 24 months with baseline values.

\section{Supplementary Files}

This is a list of supplementary files associated with this preprint. Click to download.

- Table1.xlsx

- Table2.xlsx

- Table3.xlsx

- Table4.xlsx 
- SupplementTable1.xlsx

- SupplementTable2.xlsx 